Revue d'histoire de l'enfance « irrégulière »

Le Temps de l'histoire

6 | 2004

Les sciences du psychisme et l'enfance « irrégulière »

\title{
La construction de la profession de psychologue dans le champ de la Justice des mineurs
}

Charlotte Toscani-Merle

\section{(2) OpenEdition \\ 12 Journals}

Édition électronique

URL : http://journals.openedition.org/rhei/821

DOI : $10.4000 /$ rhei.821

ISBN : 978-2-7535-1644-1

ISSN : $1777-540 \mathrm{X}$

Éditeur

Presses universitaires de Rennes

Édition imprimée

Date de publication : 15 novembre 2004

Pagination : 153-184

ISSN : 1287-2431

Référence électronique

Charlotte Toscani-Merle, "La construction de la profession de psychologue

dans le champ de la Justice des mineurs », Revue d'histoire de l'enfance «irrégulière » [En ligne], 6 |

2004, mis en ligne le 31 mai 2007, consulté le 03 décembre 2020. URL : http://

journals.openedition.org/rhei/821 ; DOI : https://doi.org/10.4000/rhei.821 


\section{La construction de la profession de psychologue dans le champ de la Justice des mineurs}

C'est parce que j'étais inscrite en psychologie à l'université de Nancy et que j'avais un cursus dans le scoutisme - éclaireuse, éclaireuse aînée, cheftaine, assistante au commissaire de province des éclaireurs de France - que je fus recrutée au centre de Han-sur-Seille, ${ }^{(2)}$ ouvert en 1947 dans le cadre de la Sauvegarde de l'enfance de Lorraine. Le commissaire de province de l'époque, un homme remarquable, m'avait alors prêté la thèse de Françoise Marette qui devait devenir célèbre sous son nom d'épouse, Françoise Dolto.

La directrice de ce centre éducatif, Yvette Resnick, avait connu la Résistance et avait rencontré quelques-unes des personnes qui avaient participé de façon plus ou moins engagée à l'aventure de l'École des cadres d'Uriage, dont Bernard Comte note « [qu'] elle présentait l'apparence mystérieuse, un peu ésotérique, de cette étrange communauté de moines-soldats-pédagogues animant un camp scout qui devient laboratoire idéologique et foyer de rébellion, inspiré par une mystique qui se prétend révolutionnaire $»{ }^{(3)}$ Pestalozzi, Montessori, Makarenko, Piaget, Freinet... et, sur un plan plus politique, Jean Zay, ${ }^{(4)}$ lui avaient fourni des éléments de réflexion pour créer une éducation nouvelle.

Elle avait conçu pour l'établissement une pédagogie, novatrice à l'époque, basée sur l'idée de self-government. Les groupes élisaient leurs déléguées, qui elles-mêmes élisaient un conseil. Une assemblée générale hebdomadaire, préparée d'une part par le conseil, d'autre part par l'ensemble des personnels, débattait des questions et projets soulevés dans la semaine. Déjà, lorsque de lourds problèmes se faisaient jour pour certains individus, il était fait appel à un psychiatre, qui pouvait assurer un suivi, mais aucun recours n'avait jusqu'alors été mis en place concernant les conflits

\section{Charlotte Toscani- Merle $^{(1)}$}

(1) Docteur en psy-

chologie.

(2) On trouvera un article d'Yvette Resnick sur ce centre in L'école publique française, avantpropos d'Albert Bayet, président de la ligue de l'enseignement, préface de P. O. Lapie, ministre de l’Éducation nationale, introduction de A. Beslais, directeur général de l'enseignement du premier degré, conclusion du président Édouard Herriot, éditions Rombaldi, Paris, 1952, p. 198-200.

(3) Bernard Comte, Une utopie combattante. L'école des cadres d'Uriage, 1940-1942, préface de

Charlotte Toscani-Merle / p. 153 à 184 
René Rémond, Paris, Fayard, 1991, p. 14.

(4) Jean Zay, 19041944, ministre de l'Éducation nationale et des Beaux-Arts au cabinet de Léon Blum en 1936. On lui doit une refonte du système éducatif, la prolongation de la scolarité de 13 à 14 ans, la création du CNRS... Il professait : «C'est l'effort d'éducation et de formation de la jeunesse qu'un gouvernement républicain considère comme sa principale préoccupation. "

(5) Michel Chauvière, «Le maréchal aimait les enfants... ", Actes, $n^{\circ} 19 / 20,1978$.

(6) Ancien élève de l'école normale supérieure de Saint-Cloud, enseignant, puis inspecteur d'académie, Henri Michard, ami d'Emmanuel Mounier, fait partie du premier groupe de institutionnels. Il lui apparut très rapidement qu'une présence "psy" dans l'établissement devenait nécessaire.

Yvette Resnick pensait également, dès cette époque, que la prise en charge des enfants et adolescents devait être le fait de personnes ayant vécu dans des centres éducatifs, donc le mieux à même de les comprendre. Dans cette optique, elle avait décroché une bourse pour la formation de l'une des adolescentes à l'école Jean-Jacques Rousseau, à Genève, dirigée par Jean Piaget (1896-1980), l'éminent théoricien de la connaissance de cette époque. Elle avait obtenu, au début des années cinquante, qu'une psychologue formée dans cette institution soit nommée à Han-sur-Seille. Cette psychologue devait assurer une fonction autre que celle d'évaluation, ce qui était tout à fait inédit.

$\mathrm{Si}$ je cite cette expérience (éphémère !) de Han-sur-Seille, c'est parce qu'elle est au carrefour d'anciens errements et de nouvelles pratiques. Elle témoigne d'un autre regard sur les enfants et adolescents et elle ouvre à des mesures éducatives originales faisant désormais une place aux processus psychiques et aux turbulences institutionnelles.

Une période charnière pour la prise en compte du sujet est celle qui a suivi la défaite de 1939, défaite qui a suscité des réflexions sur la crise $\mathrm{du}$ XIXème siècle et la révolution du XXème siècle, ayant pour but de rechercher les moyens de relever la France en enrégimentant les jeunes générations et en traitant les inadaptations. ${ }^{(5)}$

D’abord participante, sur un modèle paramilitaire, à la " révolution nationale » voulue par Pétain, puis en opposition avec elle, l'École nationale des cadres de la jeunesse d'Uriage déjà citée, créée sous l'égide du ministère de la Famille et de la Jeunesse dans le but de former des cadres pour la jeunesse, a eu un impact important. Elle a essaimé d'une part en réseau d'écoles régionales dirigées par d'anciens élèves, d'autre part, après sa fermeture, en réseau de formation pour les gens du maquis.

Les cadres de cette école étaient issus de mouvements de jeunesse florissants à cette époque. Ils avaient le souci de rassembler les connaissances, de confronter les courants de pensée, de constituer un laboratoire de recherches civiques et sociales, autant que d'expériences pédagogiques. Ils ont été pour beaucoup dans la réflexion sur les problèmes 
de l'enfance et de la jeunesse, et cela sur trois plans qui leur paraissent interférer : le droit, la pédagogie et la prise en compte de la personnalité. Ils ont eu une influence sur la mise en place "d'espaces éducatifs" où nombre de métiers auront à se trouver une juste place et un statut, en particulier celui de psychologue. Plusieurs des instructeurs ayant participé à l'expérience d'Uriage - Joffre Dumazedier, Paul Chombart de Lauwe, Bénigno Cacérès, Gilles Ferry, Olivier Hussenot... - furent appelés à enseigner au Centre d'études et de formation de l'Éducation surveillée, à Vaucresson, créé officiellement en octobre 1951 et dirigé par Henri Michard (1908-2002). ${ }^{(6)}$

La fécondité des interrogations, marquées par l'humanisme, le personnalisme, cristallise les avancées hétérogènes relatives à la prise en compte et à la compréhension du sujet et contribue à la création de la profession de psychologue. ${ }^{(7)}$ Pour ce qui concerne l'Éducation surveillée, s'amorce alors la création d'un corps de psychologues, qui se spécifie en fonction :

- des exigences d'un autre droit des mineurs et de la demande de leur "correction" impliquant un triage ${ }^{(8)}$;

- des recherches et expérimentations menées, dès avant la fin de la guerre 1939-1945, non seulement pour comprendre les enfants "normaux", mais aussi la conduite délinquante, les “irrégularités", les handicaps, les échecs scolaires et d'adaptation dont ils souffrent ;

- de nouveaux critères "scientifiques" de la psychologie, suite aux progrès relatifs à la connaissance des processus cognitifs et de la vie psychique ;

- du modèle médical longtemps prévalant ;

- de la pratique dans le champ éducatif, lui aussi se construisant.

\section{Instauration de l'usage de la psychologie dans le champ judiciaire.} Prémisses et mise en ouvre de l'ordonnance de $\mathbf{1 9 4 5}$

Une place a été faite, de tout temps, dans le droit, à l'enfant. Elle est, pour l'essentiel, relative à la responsabilité juridique, au discernement, à l'aménagement des condamnations. Le code de $1810^{(9)}$ est une révolution en ce qu'il propose la spécialisation de magistrats au clair avec les idées de juristes, d'administra-

teurs, de conseillers de

l'Éducation surveillée au

lendemain de la guerre.

Dès 1947, il joue un rôle

important dans la forma-

tion des premiers éduca-

teurs de l'Éducation sur-

veillée et des premiers

juges des enfants.

(7) La licence de psychologie est lancée, " à titre d'expérience ", par le décret $n^{\circ} 47822$ du 9 mai 1947.

(8) Le "triage" n'a pas à cette époque le sens péjoratif donné à ce jour. Il dénote l'intérêt des premiers psychologues pour la classification et le souci d'aménager une place à ceux qui se révélaient hors norme.

(9) Il n'est pas dans mon propos de mener une recherche exhaustive sur les textes relatifs aux mineurs, mais de relever les textes précisant les 
demandes de savoir, de compréhension, adressés à des spécialistes ayant compétence pour $\mathrm{y}$ répondre. Pour une étude plus complète, on peut se reporter à

Jacques Bourquin, « La longue émergence de la notion d'éducabilité du mineur de Justice ",

Revue d'histoire de la Justice, 1996.

(10) Michel Foucault, Surveiller et punir,

Paris, Gallimard, 1975, p. $300-301$.

(11) Congrès de criminologie, Stockholm, 1909. Il est à noter que les premières années du siècle sont marquées par l'intense désir, dans une large partie de la population, de revanche sur l'Allemagne et de récupération de l'AlsaceLorraine, qui aboutit, en 1912 précisément, à la loi des trois ans sur le service militaire. réforme, la création de lieux spécifiques pour le mineur (l'habitude persistait de les placer dans les mêmes prisons que les adultes avec, dans le meilleur des cas, installation dans des quartiers spéciaux) et le recrutement de spécialistes «maîtrisant les techniques de savoir et capables de dégager une appréciation exacte des faits et de la personnalité, de déterminer la capacité de relèvement et de donner l'indication appropriée à une bonne orientation ", ce qui annonce les futurs psychologues.

C'est à Mettray, dès 1840, que se concentrent toutes les techniques, parmi lesquelles la médecine, Mettray qui, " outre une fonction de dressage assumée par les techniciens du comportement, ingénieurs de la conduite, orthopédistes de l'individualité, [comprend] une fonction d'observation permanente des colons ".(10) Il s'agit de mettre en ouvre une observation s'appuyant sur les seuls critères d'objectivité scientifique, assurant le triage des enfants anormaux, délinquants, etc., triage insistant sur les valeurs de l'époque : travail, instruction "juste suffisante pour faire de bons ouvriers dotés d'un métier », discipline pour former de bons soldats. ${ }^{(1)}$ Ce dispositif fonctionne en France, avec une relative continuité, jusqu'à la deuxième guerre mondiale. ${ }^{(12)}$

La loi du 22 juillet 1912 constitue l'étape la plus importante franchie par le législateur, en ce qu'elle insiste sur l'éducation. Elle institue une juridiction spécialisée et légalise « les aménagements désirables à l'égard des enfants " : l'enquête sociale, l'examen médical, la mesure de liberté surveillée. Plus tardivement, le 15 janvier 1929, le décret portant règlement d'administration publique en exécution de cette loi fait obligation au magistrat de connaître, "bien plus que le fait matériel reproché au mineur, sa véritable personnalité, qui conditionne les mesures à prendre dans son intérêt ».

En bref, dans l'esprit du législateur, le lieu d'observation est conçu comme un instrument au service du tribunal. Il a pour fonction de mener les investigations susceptibles d'assembler les connaissances utiles aux magistrats pour décider d'une orientation. De nombreuses initiatives des associations privées, ou émergeant du cadre psychiatrique, vont relayer cette demande du judiciaire. Psychiatres, psychanalystes, criminologues se mobilisent dans l'entre-deux-guerres. 
Un tribunal pour enfants commence à fonctionner à Paris en 1914. La première thèse sur les tribunaux pour enfants est soutenue, en 1923, par Chloé Owings, assistante sociale d'une Child Guidance Clinic attachée à l'un des tribunaux pour enfants des États-Unis, qui s'était installée en France durant la guerre de 1914. C'est avec son concours, celui de Marie-Thérèse Veillot, assistante sociale, et le soutien financier de Olga Spitzer, philanthrope, qu'est créé le Service social de l'enfance, le 10 juillet 1923 . Ce service acquiert, en 1928, le château de Soulins pour en faire un centre d'observation et de triage. Initialement prévu pour effectuer des enquêtes sociales, il se dote rapidement d'une consultation " médico-psychiatrique ", puis d'une consultation d'orientation professionnelle. La présence d'un psychologue envoyé par Henri Pierron, directeur du laboratoire de psychologie de la Sorbonne, sera assurée une fois par semaine. ${ }^{(13)}$ C'est à partir de cette même année, sous l'égide d'un avocat, Henri Rollet, devenu juge spécialisé pour les affaires de mineurs, que se mettent en place les premiers services d'accueil et de triage.

Dans le même esprit, en 1925, la clinique de psychiatrie infantile est ouverte à la demande des juges du tribunal de la Seine, aux fins d'examen des enfants, sous la direction de Georges Heuyer (1884-1977) ${ }^{(14)}$ qui avait fondé la première chaire de neuropsychiatrie infantile, ouverture qui résulte d'une convention entre la faculté de médecine de Paris et le patronage de l'enfance et de l'adolescence fondé en 1890, également par Henri Rollet.

C'est dans ce lieu, émanant donc du judiciaire, que Sophie Morgenstern, l'une des premières psychanalystes d'enfant en France, avec Eugénie Sokolnicka qui a initié Françoise Dolto à la pratique du dessin d'enfant, est autorisée à mener les premières psychothérapies pour enfants en institution. Elle est assistée - ce qui est également une toute nouveauté dans ce type d'établissement - par une psychologue cantonnée toutefois aux tests. Dans le même temps, le "public" s'organise. Le ministère de la Justice crée, en 1927, un service de l'Éducation surveillée dépendant de la direction de l'Administration pénitentiaire. ${ }^{(15)}$ Il met en place des lieux d'observation qui ne seront plus directement affectés aux tribunaux et qui seront gérés par l’État.
(12) Henri Gaillac,

Les maisons de correction 1830-1945, Paris, éditions Cujas, 1971.

(13) Il est à noter que le Traité de psychologie en deux volumes - refondu en huit volumes en 19301940 -, première somme sur cette discipline, auquel ont participé tous les grands psychologues de l'époque, est sorti cette même année 1923.

(14) Georges Heuyer (1884-1977) écrit, en 1914, une thèse portant sur Enfants anormaux et délinquance juvénile.

Nécessité de l'examen psychiatrique des écoliers, où il reste fidèle à l'idée de classification et exprime le désir d'établir un "tableau" définissant, autant que faire se peut, ce que l'on appelait les irrégularités.

(15) L'Éducation surveillée devient une direction autonome du 
ministère de la Justice par ordonnance du 1er septembre 1945.

(16) Cette ville disposait, dès 1908, d'une consultation pour mineurs à la chaire de médecine.

(17) R. Meurillon (1932), Maison d'éducation surveillée de Fresnes, doc. poly., 21 pages. Publications de Vaucresson.

(18) Code de procédure civile, chap. IX sur l'autorité parentale, section II, l'assistance éducative. La loi du 4 juin 1970 vient en place de l'ordonnance du 23 décembre 1958, qui elle-même abrogeait les textes concernant la " correction paternelle", le «vagabondage ", les dispositions relatives aux " enfants maltraités ou moralement abandonnés » et aux " pupilles difficiles ou vicieux de
Les premiers centres d'observation se développeront dans les grandes villes, Marseille, Saint-Étienne, Montpellier, Lille, Versailles. Ils sont pour la plupart rattachés à des prisons, tel celui de Lyon. ${ }^{(16)}$

Un service d'observation s'ouvre pour les filles, à Fresnes, en 1927, pour les garçons en 1930, sous la direction du docteur Roubinovitch qui avait inauguré une telle pratique à la Petite Roquette en 1927, et où est menée une première expérience de service d'examens médico-psychologiques pour jeunes détenus. ${ }^{(17)}$ En 1936, une annexe médico-psychiatrique de cet établissement est placée sous la direction du docteur Marguerite Badonnel.

La guerre devait rendre ces mesures insuffisantes. À partir de l'été 1940, les locaux de la maison d'éducation surveillée de Fresnes, toujours unique tentative officielle dans le domaine de l'observation des mineurs détenus, étant surpeuplés, la création d'autres lieux s'impose. Le premier des nouveaux centres, ouvert le 1er avril 1941 rue de Crimée à Paris (dans les locaux d'un ancien pensionnat maçonnique), déterminera la date de l'abandon des dépôts ou "renfermeries » et l'adoption de l'observation, technique pour laquelle l'exercice de la «psychologie scientifique » est désormais requis.

L'ordonnance du 2 février 1945 cristallise les conceptions nouvelles sur le plan psychologique et pédagogique. Elle définit la mission du psychologue, qui est d'apporter au magistrat un « éclairage sur la personnalité du mineur » qui présente des troubles particuliers ou qui commet des actes l'amenant à être traduit en justice ou placé dans un centre d'observation :

«Le juge des enfants effectuera toutes diligences et investigations utiles pour parvenir à la manifestation de la vérité et à la connaissance de la personnalité du mineur, ainsi que des moyens appropriés à sa rééducation. $[\ldots I l]$ ordonnera un examen médical et, s'il y a lieu, un examen médicopsychologique. »

Ces obligations instaurent les modalités des interventions du psychologique et du judiciaire. Elles déterminent en quelque sorte un cadre d'instruction, en ce qu'elles donnent lieu à la constitution d'un dossier consultable au secrétariat-greffe. La fonction des psychologues s'élargira à l'assistance éducative avec l'ordonnance du 23 décembre $1958 .{ }^{(18)}$ 
Il est vite apparu aux juges des enfants que, si les centres d'observation couvraient les grandes villes, les tribunaux des villes de moindre importance, essentiellement dans les régions rurales, étaient sous-équipés en moyens. Certains de ces juges proposent au délégué permanent d'assumer en quelque sorte une fonction d'observateur itinérant. Après les expériences de Chenove et de Lyon, des initiatives se lancent (Brive, Béziers). Il s'avère que certains cas, même complexes, ne nécessitent pas une organisation aussi lourde que celle des centres d'observation, ce qui amène à souhaiter la création de consultations. Dès 1944, Daniel Lagache ${ }^{(19)}$ démontre que la consultation médico-psychologique est un organisme léger, peu onéreux, efficace. L'observation en milieu ouvert est envisagée. En 1949, à la session d'études des juges des enfants à Marly-le-Roi, Guy Sinoir propose une analyse du Rôle du psychologue dans l'observation en milieu ouvert.

À Paris, une première expérience est mise en place en 1954. N'ayant le choix qu'entre le centre d'observation de Savigny-sur-Orge et les services de la Salpêtrière, où les médecins étaient peu disposés à rédiger des rapports alors qu'ils voyaient les mineurs dans une perspective de soin, les juges des enfants, soutenus par le président Brice, avaient proposé la création d'une consultation éducative près le tribunal de la Seine.

Cette consultation, dirigée par Renée Larbaud, comprend, en 1955, sept psychologues, cinq psychiatres, un conseiller d'orientation professionnelle (les enquêtes sociales sont menées à l'époque, comme cela se pratique pour les centres d'observation, par les services sociaux de la rue du Pot-de-fer ou de la rue Montorgueil). Elle a de plus pour mission de constituer un fichier, à la disposition du tribunal pour enfants, susceptible « de fournir des informations mais aussi des éléments chiffrés indispensables à des recherches ". ${ }^{(20)}$

Comme pour les centres d'observation précédemment, cette forme de l'observation en consultation privée directement attachée aux tribunaux interpelle la politique de l'Éducation surveillée, qui ouvre, en 1957, la première consultation, celle de Nantes, bientôt suivie par celle de Lille, en janvier 1958.
l'Assistance publique ».

(19) Daniel Lagache

(1903-1972). Professeur de psychologie, il intro-

duit en France la notion de psychologie clinique. Psychanalyste, il est, dans un premier temps, inscrit à la société psychanalytique de Paris. Lors de la scission de 1953, il suit Lacan à la société française de psychanalyse. Au moment de la scission de 1963, il fonde l'association psychanalytique de France. Né dans une famille de juristes, il s'intéresse à la criminologie durant la seconde guerre mondiale. Un contact avait été pris avec lui et les professeurs de l'université de Strasbourg repliée à Clermont-Ferrand dès 1941 pour obtenir sa participation à la formation des personnels de l'Éducation surveillée. Il enrichit également le service des études, qui inscrit dans ses 
publications :

- en 1944 : Les possibi-

lités de la consultation

médico-psychologique dans

l'examen et le traitement

des jeunes délinquants,

Melun, Imprimerie

administrative.

- en 1945 : La méthode

clinique en psychologie

bumaine, Melun, Impri-

merie administrative.

- en 1946 : «Psycho-

logie clinique et délin-

quance juvénile ", Revue

de l'Éducation surveillée,

$\mathrm{n}^{\circ} 3$.

(20) Rapport du

1er janvier 1956 de

Maurice Puzin, brochure

Vaucresson 5912.

M. Puzin était alors le

secrétaire général du

centre d'orientation édu-

cative du tribunal pour

enfants de la Seine. Il

assumera les fonctions

de président de ce tribunal. On peut remarquer, dès cette date, le souci constant des magistrats, de l'administration centrale et du centre de
Vaucresson de s'associer

à toute recherche. On

retrouve ce même souci

d'associer ces instances

et les divers personnels

lors des multiples com-

missions qui se mettront

en place pour chaque

problème nouveau,

pour chaque innovation

théorique, technique

ou pratique.

(21) Rapport rédigé

par Jean Perrusset, décembre 1964.

(22) Jacques Selosse,

«Contribution à l'étude de la délinquance ", Traité de psychologie appliquée. 9. La psychologie sociale, Paris, PUF, 1972, p. $183-244$.

(23) Guy Sinoir, «Le rôle du psychologue dans l'observation en milieu ouvert ", (conférence donnée à Marly-le-
L'inscription de l'Éducation surveillée au plan sanitaire et social permet de multiplier les lieux d'accueil et d'observation en milieu ouvert. Une commission d'études, en juin 1958, envisage l'éventail des questions posées par l'ouverture de ces consultations d'orientation éducative, en particulier celui des compétences requises en vue de l'introduction de psychothérapies d'adolescents.

Malgré la dépendance plus ou moins grande aux magistrats, selon qu'elles sont privées ou publiques, les consultations suscitent progressivement les mêmes débats. Face au mineur, les psychologues peuvent-ils se cantonner à répondre à la demande du magistrat en fonction d'un savoir qui serait scientifique ou s'autorisent-ils, sinon à accomplir un travail thérapeutique, du moins à poursuivre un suivi psychologique ? Le rapport au sujet en est modifié. Il nécessite un nouvel apport théorique et une certaine éthique, d'où un questionnement de la clinique dans son rapport avec le judiciaire, qui se cristallisera plus particulièrement sur "l'écrit " : écrit pour quel dossier ? destiné à qui ? pour dire quoi ? avec quel effet ? pour quelle fin ? avec quelle considération du secret? Mais écrit, avant tout, pour transmettre quelle parole, sachant qu'il est à craindre :

- soit de ne pas "entendre " la parole d'enfants ou d'adolescents dont on sait qu'ils accèdent avec peine au verbe, et dont les mots et " l'acte sans mot » peuvent ne pas recouvrir une signification en raison de leur difficulté à se situer comme sujets d'échange ;

- soit d'être l'adresse d'une « parole sous influence ", ce qu'elle est toujours partiellement en raison du poids de la langue, de l'imaginaire social et cul- 
turel, de la position dans la généalogie infiltrée d'obligations, de conflits de loyauté et de fantasmes propres ;

- soit que, en raison de la déficience de leur milieu social et éducatif, de leur utilisation d'une autre langue, la pauvreté du vocabulaire les empêche de saisir les propos tenus et qu'ils n'aient pas les moyens de communiquer.

Le psychologue deviendrait-il un " auxiliaire de justice ", portant un " regard savant » sur les infractions judiciaires ? Devrait-il rechercher une "vérité »? Serait-il chargé de préparer l'argumentation susceptible d'asseoir la décision du magistrat : magistrat dont la "machine " a changé en fonction des idéologies du temps, des discours sur " la crise des valeurs ", modulant les représentations du punissable, de l'amendable, de l'éducable ; magistrat auquel est attribuée la charge de tenir compte de la personnalité du mineur, de comprendre l'acte commis, d'estimer l'état de dangerosité ?

Jusqu'alors, l'écrit était plutôt la chose à perfectionner. En 1964 encore, une réunion de quatre journées, animée par Guy Sinoir et regroupant une trentaine de membres du personnel de centres d'observation et de consultations publiques et privées, est consacrée à " la synthèse d'observation ", dont il faut " envisager les améliorations de forme et de fond $»{ }^{(21)}$ L'invité de marque de cette session, Roland Barthes, traite des " apports de la linguistique à l'élaboration de la synthèse et des résistances du langage à l'opération mentale de la synthèse ".

Abstraction est faite de la position du clinicien. La connotation de l'exercice de la psychologie dans le champ judiciaire est telle que certains ont pu parler de "psychologie judiciaire " ${ }^{(22)}$ ou même de "psychologues judiciaires $»{ }^{(23)}$ Ce n'est que tardivement que ce travail paradoxal, à la limite de plusieurs territoires, est interrogé. ${ }^{(24)}$ Les débats ont perduré entre ce qu'il en est de l'écoute d'une parole et le rappel du fait que les psychologues de l'Éducation surveillée participent à des obligations, mais aussi à une déontologie : la question du secret professionnel est demeurée vivace et a suscité des échanges juge/psychologue parfois acerbes.

Le problème rebondit dans les années 1990 avec les textes relatifs à l'investigation. ${ }^{(25)}$ De tels textes relancent le débat jamais clos sur la fonc-
Roi à la session des juges des enfants), Bulletin de l'Union des sociétés de patronage de France, $\mathrm{n}^{\circ} 5$, 1er trim. 1950 ; René Apellaniz et Rassa Apellaniz-Rikkers, «Étude de l'entretien clinique. À propos de l'examen psychologique des jeunes délinquants ", Rééducation, août-sept.oct. 1962, n 142-143, p. 11-62.

(24) Caroline Gillier, « Psychologie et mandat judiciaire : un paradoxe éclairant ", Cabiers de l'ANREP, 6, Les psychologues entre la tentation analytique et la prise de pouvoir institutionnelle, 1984, 1-2 ; Pierre Segond, "Sur quelques paradoxes de l'aide contrainte ", Familien. Bulletin du centre d'études de la famille, 1988, nº 7 .

(25) NOR JUS F 91 50016C du 15 février 1991, et NOR JUS F 91 50028C du 19 avril 1991. 
(26) Pierre Legendre,

Les enfants du texte.

Étude sur la fonction

parentale des États, Paris,

Fayard, 1992, p. 26.

(27) Jacques Selosse,

" Délinquance et délinquants. Réflexions sur

l'articulation entre psychologie criminelle et psychologie du judiciaire ", Bulletin de psychologie, $\mathrm{n}^{\circ}$ spécial, Comportements délictueux, XXXVI, 1982-1983, 359 , p. $209-216$. tion des psychologues dans l'espace judiciaire. Faire connaître et reconnaître l'étendue d'une fonction, qui ne saurait se limiter à la seule investigation, témoigne de ce que leur place est toujours à ajuster, par rapport et au jeune, et au judiciaire, et à l'administration, et aux institutions. Seule une articulation cohérente peut devenir structurante pour l'enfant, pour l'adolescent, à entendre dans son inscription familiale et culturelle et dans sa singularité de sujet.

Pour le clinicien, l'écrit ne recouvre pas ce qui est entendu, qui ne serait d'ailleurs qu'un premier degré, car le dire masque et révèle ce qu'il en est de la dimension de l'inconscient. Entendre pose le problème d'une double responsabilité à l'égard du sujet : comment l'amener à interroger ce qui l'a conduit, à ce moment de son histoire, dans la situation où il se trouve, et comment peser l'opportunité de ce qui est à porter à la connaissance du juge le concernant, sachant que la référence au droit peut avoir des effets cliniques, justement, par rapport à l'interdit, au sens où l'entend Pierre Legendre, son sens étymologique, un dit entre, un dit d'interposition : un dire légalement prononcé par le pouvoir en fonction, interpose son autorité, pour conduire à bon terme certaines controverses. ${ }^{(26)}$

Ainsi le magistrat tient-il la " position d'office », la fonction qui est d'aider l'enfant à se situer par rapport aux adultes, à respecter l'ordre des " places ». " Dire le droit ", intégrer l'acte dans le cadre de la loi, loi fondée sur des textes reconnus, peut avoir un effet structurant. Pierre Legendre a même pu parler de " clinique du droit ». Si bien que certains magistrats ont pu se sentir investis d'un rôle de thérapeutes, "oubliant" parfois les "faits" et l'application du droit, faisant un amalgame entre la représentation qui est la leur de la loi sociale et le désir d'approcher la réalité psychique du jeune. Pour le sujet mineur, il importe que soit prise une mesure légitimant l'acte clinique qui permette d'aménager, dans d'autres lieux, un autre trajet.

\section{Statut et pratique des psychologues à l'Éducation surveillée}

Apportant donc un "savoir" au magistrat de la jeunesse, les psychologues auront à délimiter leur place et par rapport à l'administration centrale de l'Éducation surveillée et par rapport aux personnels qui y exercent. Là encore les psychologues sont censés répondre à une deman- 
de de connaissances sur un sujet mineur au bénéfice de nombreux "autres" chargés de la régulation sociale. Il doit, le temps d'une observation, aider à l'élucidation d'un problème et à étayer une proposition en vue d'une action visant à corriger un désordre qui touche l'individu, mais aussi son écart des normes sociales. De plus, aidant à trier, il contribue à l'étude des délinquants et de la délinquance. ${ }^{(27)}$

\subsection{La mission initiale des psychologues et l'administration centrale}

L'ordonnance $\mathrm{n}^{\circ}$ 15-1966 du 1er septembre 1945, portant institution à l'administration centrale du ministère de la Justice d'une direction de l'Éducation surveillée, fait le choix d'une rupture avec les idées répressives et reconnaît la nécessité de la prise en compte des facteurs psychiques de l'individu.

En 1942-1943, la seule compétence reconnue étant celle des psychiatres, la question s'est posée de leur confier la direction des centres d'observation. Le docteur Badonnel avait été pressentie, mais l'administration opta pour une direction éducative. Une exception pour le centre de Savigny, dirigé un temps par Guy Sinoir. Parmi ces spécialistes ayant pour fonction d'accueillir et d'orienter des enfants et adolescents, les " assistants de psychologie » ${ }^{(28)}$ furent recrutés.

L'une des premières à frayer le chemin de la psychologie dans l'Éducation surveillée en tant qu'assistante de psychologie contractuelle fut Rassa Rikkers (1914-2000). Elle est recrutée, le 15 juillet 1943, dans l'un des centres d'accueil et de triage dépendant du tribunal pour enfants et adolescents de la Seine, celui de Villejuif, où elle est reçue par une psychologue, Ruth Pruschy, qui deviendra Madame Lagache. Elle est nommée en 1945 au centre d'observation de Savigny-sur-Orge. Comme de coutume à l'époque, elle a entrepris une licence de philosophie, a reçu l'enseignement de l'institut de psychologie attaché alors à l'École des hautes études, puis celui de l'Institut national d'orientation professionnelle.

Guy Sinoir (1902-1978) a une formation de conseiller d'orientation professionnelle. ${ }^{(2)}$ Déjà il se déclare à la recherche d'attitudes cliniques face aux tenants de la psychologie de laboratoire. ${ }^{(30)}$ Mais il n'est pas intéressé par la gestion administrative. Il est remplacé au bout de

(28) Cette expression avait encore cours dans les années cinquante : au IIème congrès de l'UNAR, le rapporteur de la deuxième commission, M. Rey, estime que

« l'appellation d'assistant de psychologie a l'inconvénient de subordonner la qualification professionnelle (psychologue) à une fonction (assistant) qu'elle déborde ». Le congrès propose de lui substituer celle de "psychologue, assistant près d'un service de... » (Rééducation, nov. 1950). Voir supra, note 7.

(29) Guy Sinoir,

\section{L'orientation}

professionnelle, PUF, Que sais-je, 1943.

(30) Guy Sinoir,

« Nature et fonctionnement du centre d'observation de mineurs délinquants ", Sauvons l'enfan$c e, \mathrm{n}^{\circ} 65$, juillet-août 1946 , p. $1-4, n^{\circ} 66$, septoct. 1946. 
(31) Maurice Debesse, qui a écrit, en 1941, La crise d'originalité juvénile, puis L'adolescence, a fait partie du comité scientifique du service de la recherche de Vaucresson, de 1964 à 1972.

(32) Celle-ci ouvre, en 1947, le centre de Brécourt, considéré à l'époque comme une avancée révolutionnaire sur le plan de l'éducation des filles, au même titre que celui de Hansur-Seille.

(33) Paul Lutz, « Ma rencontre avec Aichhorn ", Rééducation, $n^{\circ} 254$, juin 1973 , p. 1-7

(34) August Aichhorn, Verwabrloste Jugend (1925), traduction française Jeunesse à l'abandon, préface de Sigmund Freud, Toulouse, Privat, 1973.

(35) Pierre Ceccaldi (1910-1969) fut, pendant la guerre de 1939-1945, quelques mois dans ce poste de direction. Il devient inspecteur à l'administration centrale. Il se charge alors, jusqu'à sa retraite, de la sélection des personnels et de l'élaboration de la psychologie à l'Éducation surveillée.

Paul Lutz, magistrat, inspecteur puis sous-directeur à l'Éducation surveillée où il œuvre jusqu'à sa retraite, très en avance sur son temps, est également très favorable à une ouverture vers la clinique. Il est porteur d'une autre vision de l'adolescence, objet de nombreuses recherches à cette époque. ${ }^{(31)} \mathrm{Il}$ est intéressé par la criminologie et a des conceptions ouvertes de l'éducation.

Il est un ami de Dominique Rielh, ${ }^{(32)}$ psychologue, qui, après avoir exercé à l'institut Oberlin, en Alsace, est devenue l'assistante de Daniel Lagache, repliée comme lui avec les professeurs de l'université de Strasbourg à Clermont-Ferrand. Il a rencontré les pionniers de l'époque, dont Aichhorn ${ }^{(33)}$ qui s'inspirait des théories freudiennes dans la méthodologie éducative des adolescents en difficulté. ${ }^{(34)}$

Pierre Ceccaldi, ${ }^{(3)}$ Paul Lutz, Henri Michard, Guy Sinoir ont une influence décisive sur les orientations de l'Éducation surveillée. Dans la conjoncture de l'après-guerre, ils sont les acteurs d'une transformation fondamentale des politiques institutionnelles relatives aux mineurs. Il ont le souci de faire appel à des personnels compétents et veillent à leur obtenir un statut.

En effet, à son arrivée à l'Éducation surveillée, le psychologue, attaché au laboratoire des tests, assistant, est classé, dans la rubrique des personnels, au nombre des auxiliaires, comme le jardinier, le concierge, l'aumônier... Ils auront beaucoup à faire pour que leur métier soit reconnu. J. Pinatel, ${ }^{(36)}$ lors d'une inspection au centre de la rue de Crimée, en 1944, bien que manifestant ses doutes sur l'utilité des tests, suggérait une "interpénétration étroite des services psychologiques et pédagogiques [...] qui devrait permettre d'une part de faire profiter les psychologues des leçons de l'expérience et du bon sens, d'autre part d'éviter aux éducateurs de céder à l'empirisme ".

Il est vrai qu'aucune des deux professions n'est encore définie. Celle d'éducateur ne le sera qu'avec l'institution d'un concours et d'une for- 
mation spécifiques, organisés pour la première fois sous la direction de Henri Michard au centre de Vaucresson en 1951, et avec le statut provisoire de 1945, entériné, une dizaine d'années plus tard, par le décret $\mathrm{n}^{\circ} 56-398$ du 23 avril 1956. Les articles 4 et 5 de celui-ci, abrogés par le décret $n^{\circ}$ 89-60 du 30 janvier 1989, autorisent les éducateurs ayant les compétences requises à exercer en tant que psychologues. Les premiers psychologues conserveront, jusqu'en 1981, les dispositions de ce statut du personnel éducatif qui leur permet de briguer, éventuellement, une direction d'établissement. Mais la plupart des psychologues bénéficient, dès le début, d'un engagement sous contrat.

Le cadre de leur fonction sera fixé, en 1949, par les Instructions provisoires sur l'examen psychologique (dix pages dactylographiées), signées par le garde des Sceaux, ministre de la Justice. Ces instructions ont été préparées, sous la direction de Guy Sinoir, par une commission « qui comprenait des spécialistes des différents postes » d'observation. Elles prévoient, dans les moindres détails, le fonctionnement, l'organisation matérielle, l'élaboration des méthodes, le choix et l'application des épreuves, la présentation des résultats, les liaisons avec les autres services.

Psychologues “de” l'Éducation surveillée, donc ! C'est ce que stipule le tardif décret $\mathrm{n}^{\circ} 81-243$ du 12 mars 1981 portant statut particulier des psychologues des services extérieurs de l'Éducation surveillée, statut qui les classe en catégorie A de la fonction publique. Après avoir soulevé nombre d'interrogations sur les orientations de l'administration centrale, ce statut a été finalement souhaité. Il a été précédé par de nombreuses prospections. Une enquête sur les services de psychologie, lancée en 1965 par Guy Sinoir et son assistante Suzanne Lamarque, porte essentiellement sur le désir d'une harmonisation de la profession.

Certains psychologues, mandatés par leurs collègues, assistent, dès sa création en 1965, aux réunions du CCOP (comité de coordination des organisations de psychologues) animé par Didier Anzieu. ${ }^{(37)}$ Un sondage, en 1968, obtient majoritairement un assentiment sur le rattachement à la fonction publique, associé de conditions : possibilité de maintenir un travail à l'extérieur, autonomie, exercice à mi-temps, statut de psychothérapeute à définir... responsable du service de l'Éducation surveillée qui dépendait alors de la direction pénitentiaire. Il y fut rejoint en 1941 par Paul Lutz. L'idée s'imposa à eux que les problèmes des mineurs ne pouvaient être traités dans ce cadre. À la

Libération, il est nommé sous-directeur de la nouvelle direction de l'Éducation surveillée, dont il devient le directeur de 1956 à 1964.

(36) Jean Pinatel est inspecteur général de l'administration centrale. Il a été secrétaire général de la Société internationale de criminologie de 1950 à 1965, puis président de la commission scientifique de cette société. En 1973, il en devient le président. Son œuvre est inspirée par les thèses de De Greeff.

(37) Didier Anzieu (1923-1999) devient l'élève de Lagache en 
préparant l'agrégation de philosophie. Il est ensuite son assistant à la Sorbonne. Psychanalyste, il suit Lagache lors des scissions. Il est l'un des fondateurs de l'Association psychanalytique de France. Il devient un spécialiste des méthodes projectives, du psychodrame, de la dynamique de groupe. Il est l'auteur d'ouvrages faisant référence et dirige, aux éditions Dunod, les collections traitant de psychanalyse et de psychologie clinique.

(38) Jacques Selosse (1923-1995), professeur à l'université de Lille III, chargé de cours à l'institut de psychologie de Paris V, a dirigé le Centre de formation et de recherche de Vaucresson, après le départ de Henri Michard, jusqu'en 1980.

(39) À noter qu'il y
Ces démarches des psychologues de l'Éducation surveillée sont rendues plus aiguës encore du fait du départ à la retraite de Guy Sinoir, fin 1967. En effet, Guy Sinoir centralisait les questions et son autorité auprès de l'administration centrale restait la seule caution sérieuse. Un projet d'organisation de la profession des psychologues à l'Éducation surveillée, longuement discuté, est déposé au ministère le 12 février 1968.

Ce n'est qu'à la suite du décret de 1981 qu'une commission présidée par Jacques Selosse ${ }^{(38)}$ reprend l'étude des conditions d'exercice de la profession qui seront soumises à un comité technique paritaire. Elle aboutit, le 31 mars 1983, à la note portant sur l'application de ce décret signée par Myriam Ezratty, magistrate, alors directrice de l'Éducation surveillée. Elle officialise l'autonomie totale dont les psychologues bénéficient, depuis les années soixante, pour le choix des techniques qui leur paraissent les plus appropriées compte tenu des théories auxquelles ils désirent se référer.

La promulgation de la loi du 25 juillet 1985 sur l'usage professionnel $\mathrm{du}$ titre de psychologue et l'inscription dans un corps de psychologues devait asseoir la profession dans son ensemble. Le décret 96-158 du 29 février 1996 crée enfin un statut particulier du corps des psychologues de la Protection judiciaire de la jeunesse, ce qui a demandé cinquante ans!

\subsection{La pratique dans les centres d'observation et les consultations}

En 1951, le centre d'observation de Savigny-sur-Orge, ayant regroupé au fil des mois les centres d'accueil et de triage de la Seine créés en 1942-1943 dans la région parisienne - le centre de Villejuif (dans un quartier isolé de l'hôpital psychiatrique), le centre de Charenton (dans le Fort), le centre de la rue de Madrid à Paris (dans un ancien internat des Pères jésuites), l'établissement des Tourelles (dans un quartier spécial de la caserne) - devient le lieu de la production de la connaissance du jeune délinquant, grâce à une organisation fondée sur le modèle préconisé de l'observation "scientifique".

Au moment de l'ouverture du centre d'observation de Bures-sur-Yvette, en 1958, ils ne sont encore que dix pour toute la France. L'ouverture des consultations élève progressivement leur nombre. Quelques chiffres 
apparaissent au hasard, les archives de l'Éducation surveillée ne disposant pas de statistiques annuelles régulières avant 1976. Guy Trembelland fait état, dans le Bulletin syndical, le 17 juin 1954, de 10 psychologues : 3 sur statut éducatif, 7 sur contrat. "Nous serons 28 psychologues ", écrit René Apellaniz, le 1er février 1962, à un collègue. En décembre 1963, un questionnaire de préparation pour une journée nationale d'études à Vaucresson a été envoyé aux 41 psychologues en poste. En novembre 1965, ils sont 50. En octobre 1967, 60 psychologues se répartissent dans 31 services dispersés sur tout le territoire. Au moment de l'intégration dans le corps des psychologues, en 1981, ils sont $151 .^{(39)}$

Malgré quelques interdits, tel celui de rencontrer les familles, ils acquièrent une relative indépendance quant à leur démarche professionnelle. Ils choisissent leur méthode d'approche en refusant à ce niveau toute ingérence. Cependant, les quotas qui leur sont imposés privilégient le rendement au détriment du qualitatif (le centre d'observation de Savigny-sur-Orge reçoit, les premières années, 800 à 900 adolescents ; certaines consultations se voient confier 200 à 300 cas). ${ }^{(40)}$

Le service de psychologie dispose d'un véritable laboratoire où " règne la psychologie comme science nouvelle, accompagnée d'un certain fétichisme des tests, des instruments, des étalonnages ", ainsi que le raconte Yann Malefant, l'un des premiers psychologues. L'approche clinique n'interroge pas la finalité, les règles, le rite de l'examen psychologique. Vêtus de blouses blanches, disposant de bureaux à l'écart des autres personnels, ils utilisent sensiblement les mêmes batteries de tests :

- tests d'intelligence, sur le plan "verbal" : Binet-Simon, TermanMerril $^{(41)}$; sur le plan "non verbal” : Vermeylen, Decroly...

- d'ordre morpho-psychologique, psychomoteur, d'intelligence pratique : Baille, Stenquist, Carrard, Kohs, Piorkowski, Rey...

Sont également utilisés : un questionnaire d'analyse de caractère, les tests de barrage de Pressey, les tests de sens éthique de May et Hartshome, et les tests de Woodworth et Mathews sur les tendances émotives, psychasthéniques, schizoïdes, paranoïaques, hypocondriaques, impulsives, instables, perverses, tous tests qui avaient été modifiés par Georges Heuyer. Beaucoup de ces tests n'ont plus cours, mais leur nombre indique bien le avait (rapport de 1953) une femme pour quatre hommes. Les chiffres s'inversent ensuite. Les hommes sont désormais une petite minorité.

(40) Voir le témoignage de Odile Duchier, psychologue à Savigny de 1960 à 1997, Ma ferme de Champagne - Mémoires d'bumeur et d'bumour d'une psychologue, Association pour l'histoire de l'Éducation surveillée et de la protection judiciaire des mineurs, Vaucresson, 1999.

(41) Le Terman, épreuve de niveau intellectuel, a été remplacé progressivement à la fin des années soixante par le Wechsler-Bellevue, qui permet d'obtenir des informations plus fines sur le fonctionnement intellectuel des adolescents, en particulier dans sa partie performances et qui donne des indications 
sur les modes de réaction du sujet.

(42) Rassa ApellanizRikkers, René Apellaniz, "Exposé d'une méthode d'examen psychologique adapté aux troubles de l'adolescent ", 19551957, in Charlotte Toscani-Merle, Nicole Vacher, Odile Duchier, Daniel Fleury, MarieThérèse Mazerol, Ariane Casanova, Yves Gazzera, «Approche historique de la clinique à l'Éducation surveillée ", Cabiers $d u$ CRIV, Vaucresson, 1990.

(43) Pierre Mâle (19001976), psychiatre, est l'un des premiers membres de la société de psychanalyse de Paris en 1932. Il dirige le service de guidance infanto-juvénile à l'hôpital Henri Rousselle. Il a souci d'un apport "scientifique", qui devait renseigner sur toutes les facettes d'un “être”. Déjà on reconnaît la valeur des moyens d'expression tels le jeu, le dessin, l'autobiographie, les contes libres, le mime...

Ces tests, les psychologues les modifient, les adaptent à la population, quand ils n'en créent pas. Rassa Apellaniz-Rikkers expérimente le TPGA (test de projection graphique) et le TESO (test d'étude sujet/objet). Parallèlement, avec son mari, elle forme à l'entretien qui prend une place de plus en plus centrale. Elle essaie de théoriser la pratique. ${ }^{(42)}$

Les tests projectifs commencent à être utilisés, en particulier le Rorschach qui paraît à beaucoup une avancée importante, le TAT (Thematic Aperception Test), le MMPI (inventaire multiphasique de personnalité du Minnesota), le test de l'arbre... Marie-Thérèse Mazerol mène une recherche sur le test du village.

Les conclusions donnent lieu à "réunions de synthèse". Pour le centre d'observation de Savigny, "modèle" pour les autres centres, s'organisent, le mercredi, deux types de réunions :

- une réunion d'information et d'orientation technique ;

- une réunion de synthèse, après sept ou dix semaines selon la difficulté à réunir informations et examens. Elle donne lieu à un partage des éléments recueillis, à une confrontation des points de vue entre les divers partenaires et à la recherche d'une proposition de mesure. La conclusion du psychologue comporte la nomenclature des tests, l'exposé des faits ayant justifié l'ordonnance du magistrat, la situation familiale et l'histoire de l'adolescent, les ressources intellectuelles et affectives, une approche de la structure et se termine sur une hypothèse relative à l'évolution du cas et sur la discussion des mesures à envisager. Les conclusions du psychologue et du psychiatre sont jointes in extenso au rapport final, rédigé en premier lieu par Rassa Rikkers, puis par le chef de service éducatif ou l'éducateur. Ainsi, la synthèse reste en fait une juxtaposition de paragraphes parfois sans réelle cohérence quant à la prise en compte du fonctionnement psychique du mineur et à son poids sur les propositions de mesures. Un chef de service éducatif, muté du centre d'observation de Lyon où la procédure était différente et ayant tenté d'incorporer dans le rapport d'observation des "morceaux choisis" des examens psycholo- 
gique et psychiatrique, s'est vu convoqué par les psychologues et le docteur Mâle, ${ }^{(43)}$ et son expérience ne s'est pas poursuivie.

Les psychologues font pratiquement tous leur stage de candidature à Savigny-sur-Orge qui compte trois, puis quatre psychologues. Ils exercent quasi exclusivement dans les centres d'observation jusqu'au début des années soixante. C'est dire que beaucoup d'entre eux se connaissent et qu'il existe une relative harmonisation des pratiques. Ils gardent un dessein unifié de leur fonction et commencent à représenter une autorité fondée sur l'idée qu'ils détiennent un savoir spécifique non négligeable. Sous la direction de Guy Sinoir, psychologues du public et du privé réunis échangent sur leur pratique et se regroupent lors de stages.

Devant cet avènement de la clinique à l'Éducation surveillée, l'administration centrale ne se montre pas sans réticences. Pierre Ceccaldi reste peu disposé à laisser la psychiatrie et la psychanalyse prendre une trop grande emprise, mais Paul Lutz continue à soutenir cette évolution.

Malgré ses réserves, Pierre Ceccaldi propose, en mai 1949, un stage de perfectionnement des observateurs (éducateurs et psychologues, qui se voient alors mis sur le même plan) à Marly-le-Roi. Dès ce moment, on peut noter une diversité des apports théoriques, qui se maintiendra au long des années. Par exemple, ce stage présente une "introduction à la psychanalyse ", par Guy Sinoir. Daniel Lagache parle de psychanalyse et délinquance, Paul-René Bize de " morphologie humaine et observation ", Henri Michard des principes de l'observation, François Dhallenne, directeur du centre d'observation de Savigny-sur-Orge, de l'organisation d'un tel établissement, et Pierre Mâle de "l'observation psychiatrique en centre d'observation ».

En juillet 1952, un stage réservé aux psychologues étudie plus particulièrement les quatre points suivants qui éclairent sur les options du moment : profil psychologique, épreuves de base de l'examen psychologique (ces deux points avec un souci de normalisation déclaré), test de projection du délinquant, rapport d'examen psychologique.

Un second stage se déroule à Vaucresson en octobre 1957. Préparé par une enquête et des groupes d'études, il porte sur l'étude d'un type de personnalité délinquante, le "fruste ", et sur la collaboration des psychologues à la formation des éducateurs. Cette session sur le fruste ${ }^{(44)}$ assuré des vacations tous les vendredis au centre d'observation de

Savigny de 1946 à 1976.

Il y recevait les cas les

plus complexes, ceux

pour lesquels se posaient les problèmes d'indica-

tion de soin, de psychothérapie... Il échangeait avec les psychologues qui assistaient à ses examens. Il les "écoutait" et dictait des conclusions qui étaient toujours “simples”. Il a participé aux travaux de recherche et aux stages de formation du centre de Vaucresson.

(44) La notion de frusticité chez l'adolescent délinquant, Extraits des travaux de la Irème session des psychologues praticiens de la délinquance juvénile, rédigés par Guy Sinoir et Guy

Trembelland, assistés de Mmes Gilles et Mazerol, de MM. Bournonville, Casalis, Hyrien, Vaucresson, 1959. 
(dont il semblait que le terme avait une forme "mal définie » et correspondait à une entité "mal systématisée d'adolescents " qui ne "saurait être assimilée à la débilité mentale » mais à " une structure floue ") est intéressante en ce sens qu'elle marque la perplexité toujours vivace des cliniciens face à ces jeunes dont les comportements "irréductibles " déconcertent et dont les positions par rapport à la loi, au temps, à la mort, à l'autre, ne peuvent être renvoyées à des structures précises.

Guy Trembelland tiendra dans cette session une place importante. Il exerce en province. Après avoir participé à l'ouverture du service de psychologie du centre d'observation de Collonges, près de Lyon, en 1949, il assume depuis 1954 les fonctions de chef du service de psychologie de Marseille. Comme ses collègues parisiens, il a le souci de rencontrer d'autres psychologues et crée dans chacune de ces villes une association. Celle de Marseille - la société de psychologie du Sud-Est, première société régionale - fusionnera par la suite avec la société française de psychologie. Elle organise des échanges, des conférences, auxquels apportèrent leur concours, entre autres, Simon, Piaget, Lacan, Baudoin, Hesnard.

\subsection{Les psychologues et les psychiatres}

Cette coexistence de théories diverses, si elle ne semble pas gêner, dans la pratique, une certaine cohérence de l'ensemble, soulève quelques tiraillements. Les relations entre les psychologues et les psychiatres se nouent différemment selon leur fonction affichée : chercheurs, médecins-psychiatres, psychiatres-psychanalystes.

L'image du délinquant ou du jeune handicapé est d'abord fortement connotée par la maladie mentale. Alexis Danan cite, dans Paris soir du 10 janvier 1937, l'expérience du « camion psychiatrique » à l'usage des délinquants mineurs, qui devait leur éviter l'envoi en cellule :

"Mais on découvrit tant de demi-fous, tant d'épileptiques, tant d'hérédo-alcooliques manifestes dans les quartiers disciplinaires d'Eysse, d'Aniane, et même dans ceux de Belle-Ile, de Lamotte-Beuvron, de Clermont, de Doullens, qu'il aurait fallu dix laboratoires mobiles pour répondre à tous les besoins. » 
Les lieux d'observation ont bien évidemment leur médecin-psychiatre attitré ; certaines colonies, maisons de correction et d'éducation surveillée aussi. En 1939, à Saint-Hilaire, des consultations médico-psychologiques avaient été estimées assez indispensables pour être reprises, après une interruption due à la guerre, en 1941. Il semble à tous naturel que l'abord des troubles mentaux soit réservé aux psychiatres. Toutefois l'administration centrale - Paul Lutz en particulier - souhaite déjà doter chaque établissement d'éducation d'un psychologue qui aiderait à la compréhension des "problèmes ». Déjà la psychanalyse soulève une « révolution dans la manière de penser les problèmes qui jusquelà avait été traités par la psychiatrie ». ${ }^{(45)}$

D'ailleurs les options des psychiatres eux-mêmes sont différentes. Ils ne se veulent pas tous cliniciens, mais "scientifiques", tel le docteur Bize, neuropsychiatre, tenant de la biotypologie. Il fait partie de la première équipe des enseignants du centre d'études et formation de Vaucresson. Il est également conseiller technique à l'Éducation surveillée. Chercheur au Conservatoire des arts et métiers, il a été président, de 1952 à 1957, de l'ARERAM, dont il fut le fondateur avec Simone Ramain, bien connue pour ses méthodes d'apprentissage. Ses options répondent aux préoccupations des acteurs de l'époque, pour lesquels le travail est une valeur rédemptrice.

Il a par ailleurs inauguré la revue les Annales de Vaucresson, avec une "Étude comparée de la psycho-motricité des mineurs d'un centre d'observation et de diverses populations de non-délinquants ». Il poursuit ses recherches durant plusieurs années au centre d'observation de Savigny-sur-Orge et, s'il professe la psychologie scientifique, il n'interfère jamais sur le service. De même, le docteur Verdeaux, attachée au CNO de Fresnes, effectuera, au début des années soixante, dans ce même centre, une recherche sur les EEG d'adolescents délinquants. Il s'agit bien, pour l'un comme pour l'autre, de contribuer à la connaissance de la population particulière qu'ils étudient.

C'est dans l'institution d'observation que les compétences techniques des psychologues semblent constituer une menace pour certains psychiatres. En effet, la fréquentation des lieux de formation à la clinique -
(45) André Green,

"Sur l'état de santé de la psychanalyse aujourd'hui " et " La psychanalyse est-elle mortelle?", in Cent ans après, entretiens de Patrick Froté avec J.-L. Donnet, A. Green, J. Laplanche, J.Cl. Lavie, M. de M’Uzan, J.-B. Pontalis, J.-P. Valabrega, D. Widlöcher, Gallimard, coll. l'Inconscient, Paris, 1998, p. 96-167. 
séminaires, présentations de cas dans les hôpitaux psychiatriques, conférences de l'institut de psychanalyse, groupes de contrôle - amène les psychologues à envisager leur profession sous un angle nouveau et estompe leur position de faiblesse face aux psychiatres. De plus, le choix leur est laissé de peser l'indication de l'examen psychiatrique pour les enfants et adolescents. La profession des psychiatres est pourtant assise de longue date. Ils appartiennent à un corps régi par un conseil de l'Ordre. La rédaction d'un diagnostic, d'un pronostic, l'ordonnance d'un soin relatif aux troubles leur incombe. Ils peuvent exercer la fonction d'expert auprès des tribunaux.

Pourtant l'autorité du médical leur parait interpellée à tel point que cela donne lieu à débats passionnés. À la suite d'une circulaire du garde des Sceaux ( $n^{\circ} 5072$ en date du 9 septembre 1950), sur les « conditions d'utilisation distincte par les magistrats des tribunaux pour enfants des examens médico-psychologiques et psychiatriques ", dont l'initiative revenait à Guy Sinoir, les psychiatres se sont alarmés et, après avoir été saisis par le docteur Crémieux de Marseille, ils se réunissent en commission à Sainte-Anne sous l'égide du professeur Heuyer.

Le procès-verbal de cette commission, dans sa séance du 27 janvier 1953, fait état d'une documentation relative aux réglementations en vigueur à l'étranger (États-Unis, Suisse, Belgique, Pays-Bas, Danemark, Grande-Bretagne...) réunie par les docteurs Daumezon et Ey. S'appuyant sur le fait que le diagnostic est un des éléments spécifiques de l'activité médicale, ils voient dans cette circulaire une invitation pour les nonmédecins à l'exercice illégal de cette profession.

Ils y perçoivent un problème doctrinal et trouvent déplaisant pour les représentants d'une discipline d'entamer avec une discipline adverse une dispute. Ils craignent l'extension des pratiques des psychologues aux adultes. Ils craignent aussi de perdre leur place auprès des juges. Ils concluent :

«a) la circulaire du 9 septembre 1950, dans la mesure où elle amène le juge à interpréter seul les données d'un examen psychologique, à faire seul la synthèse entre les résultats de cet examen et ceux du médecin peut être une source de graves erreurs préjudiciables aux enfants. 
b) une conception purement psychologique de ce comportement (inadapté) [est $]$ en contradiction avec les données les plus sérieuses de la science psychologique inséparable de la biologie.»

Ils demandent l'avis du conseil de l'Ordre avant de soumettre une intervention au garde des Sceaux. ${ }^{(46)}$

Le même débat autour de l'appellation des CMPP a eu lieu : alors que le premier centre psychopédagogique, le centre Claude Bernard, a été, à son ouverture en 1946, très largement inspiré par la psychanalyse, il a été obligé de s'adjoindre le mot "médico" - centre médico-psycho-pédagogique - afin de satisfaire aux exigences des pouvoirs publics.

Pourtant, durant de nombreuses années, après le départ de Sinoir, les médecins-psychiatres furent seuls interlocuteurs à l'administration centrale en tant que conseillers techniques pour les cliniciens.

Le docteur Certhoux, qui organisait un colloque annuel pour les médecins à Vaucresson, l'ouvrit progressivement à quelques psychologues. En 1986, à l'arrivée de Maryse Vaillant au service des études, il le transforme en journées des cliniciens de l'Éducation surveillée, journées qui ne firent pas l'économie de polémiques entre psychiatres et psychologues. Plus les candidatures de psychologues, puis d'infirmières, pour ce colloque augmentent, plus le nombre de médecins et de psychiatres diminue.

Peut-on penser que, dans les débuts, persiste, pour les décideurs, l'idée sous-jacente que le psychologue demeure le pourvoyeur d'un examen utile au magistrat et aux éducateurs, mais qu'il existe un certain sentiment de hiérarchisation entre les différents protagonistes, générateur de conflits? Ou bien que, des cadres conceptuels ne s'étant pas mis en place, il se soit produit, sous le mot clinique, un certain amalgame concernant les praticiens "psy" ?

\subsection{L'adresse à la psychanalyse}

L'intérêt pour la théorie psychanalytique a été constant à l'Éducation surveillée tout au long de la mise en place de nouveaux modes de compréhension de l'enfant et de l'adolescent. On a vu que Paul Lutz connaissait Aichhorn. Celui-ci, dans un recueil de conférences dont la pré-
(46) S'étaient expri-

més, à cette séance du

27 janvier 1953, les docteurs Abely, Daumezon, Ey, Minkowski, Gallot, Figon, Belley, Le Guillant, Dublineau, Bonnafé, Aujaleu... 
(47) Kate Friedlander,

La délinquance juvénile Théorie - Observation Traitement, 1945, traduit par Anne Berman, 1951, PUF, Bibliothèque de psychanalyse et de psychologie clinique.

(48) Assistaient à ce premier stage ceux qui se comptaient parmi les premiers psychologues de l'Éducation surveillée : Joseph Villier, Guy Trembelland, Yann Malefant, Renée Gautheron-Larbaud, René Apellaniz qui allait épouser Rassa Rikkers. face par Freud faisait état des trois métiers impossibles - éduquer, guérir, gouverner -, témoignait des résultats d'une expérience de rééducation s'inspirant de la psychanalyse comme nouveau mode d'appréhension des processus psychiques.

D'autres recherches, à partir des théories freudiennes, sur la population des jeunes délinquants commencent à avoir un fort impact, en particulier celle de l'école anglaise. Kate Friedlander ${ }^{(47)}$ sera beaucoup lue dans les années cinquante, de même que Mélanie Klein et, plus tard, Winnicott qui "travaillent" de façon originale « la consultation thérapeutique des enfants » et des jeunes délinquants.

Les premiers psychanalystes manifestent un vif intérêt pour la criminologie. La Revue française de psychanalyse, périodique de la société psychanalytique de Paris, publie dans son premier numéro, en 1927, l'article de Marie Bonaparte sur "Le cas de Madame Lefevre ", réquisitoire pour un traitement des criminels et pour la création d'asiles-prisons. Jacques Lacan effectue sa recherche sur les sœurs Papin en 1933. En collaboration avec Michel Cénac, il présente sa communication «Introduction théorique aux fonctions de la psychanalyse en criminologie " à la XIIIème conférence des psychanalystes de langue française, le 29 mai 1950, à laquelle assistaient Lagache, Lebovici (dont les présentations d'enfants dans le XIIIème arrondissement de Paris furent suivies par certains psychologues de l'Éducation surveillée), Pasche, etc.

Au centre de Villejuif, Rassa Rikkers rencontre Daniel Lagache qui, après avoir animé un stage de formation en $1943,{ }^{(48)}$ va continuer à exercer un grand rôle dans l'enseignement et les études au centre d'études et de formation de l'Éducation surveillée.

C'est à l'INOP qu'elle rencontre Pierre Mâle, qui assure des vacations au centre d'observation de Savigny-sur-Orge de 1946 à 1976. De même, jusqu'à sa retraite dans les années soixante, le docteur Badonnel, qui est perçue comme une psychiatre "classique" (portant blouse blanche, mise, durant son absence, sous une housse dans le bureau qu'elle utilisait), avec lequel les psychologues se sentent dans une position d'assistants. Il n'y avait pas d'échanges. Avec Pierre Mâle s'amorce un tournant, tracé par le mouvement culturel de l'époque et l'intérêt progressif pour les décou- 
vertes freudiennes. Remarquable enseignant à partir de la pratique, il instaure d'autres modes de travail ; les psychologues peuvent discuter sur les cas avec lui. Pour eux, s'ouvrent d'autres mises en perspective.

La dimension thérapeutique est dès lors importante pour tous les psychologues, bien qu'ils s'interrogent sur les implications d'une " attitude analytique » dans le cadre judiciaire et travaillent sur les notions de loi/Loi, de langage, d'acte, de lien, de demande, de travail dans ou sur le transfert, de l'opportunité d'une approche thérapeutique dans ce temps de la contrainte... Ce qu'ils ressentent, c'est que l'on relativise le testing, que l'on cesse de se cantonner à la formulation d'un écrit et que l'on tente de prendre autrement en compte la souffrance des enfants et adolescents, de même que celle de leur famille.

Il ne s'agit pas pour autant de travailler en tant qu'analyste, mais dans le prolongement de la psychanalyse, avec des objectifs et des moyens appropriés. Bien que les méthodes y restent assez classiques, l'adoption de ces nouvelles conceptualisations du travail des psychologues aboutit à la création par Rassa Apellaniz-Rikkers, en 1963-1964, de la consultation spécialisée d'orientation éducative, rue de l'Arbre sec à Paris (transférée à Levallois en 1976), dont les méthodes prennent appui sur une psychologie à dimension analytique. Exceptionnellement, ce lieu comprend trois psychologues et autant de psychiatres-psychanalystes. Son projet est de s'occuper plus spécialement de la population dont les troubles d'insertion dans les milieux marginaux, en même temps que les échecs éducatifs, permettent de penser qu'elle véhicule la délinquance juvénile. Les jeunes usant de drogues sont acceptés.

Cette consultation avait été envisagée à la suite d'une étude de Rassa Apellaniz-Rikkers (rapport du 4 janvier 1964) portant sur 1880 dossiers représentant l'ensemble des cas examinés dans les établissements de la Seine et de la Seine-et-Oise en 1961. Sur ce chiffre, 423 demandes de thérapie avaient été formulées à la suite des réunions de synthèse, avec une proportion de trois-quarts lorsqu'une mesure de milieu ouvert était préconisée. Les magistrats associés à ce travail libellaient ainsi leur ordonnance, afin de faciliter la visée thérapeutique : "Le psychologue commis devra, en outre, amener toute la suite psychologique souhaitable. » 
D'autres consultations s'ouvriront - en particulier celle de Versailles, animée par Joseph Villier - qui comporteront la même référence théorique et le suivi effectué par les cliniciens. L'accent y sera mis sur la formation des personnels éducatifs et sur la supervision des équipes, afin de les rendre attentifs au sens de l'acte de délinquance, au sens de la conduite de rupture et au sens de l'action à promouvoir. Toutefois le problème de la thérapie, non demandée par un patient, ordonnée dans ce contexte à un mineur, "pour son bien », ne va pas tarder à être posé avec vigilance.

\subsection{Les psychologues et les personnels éducatifs}

Les psychologues qui font leurs premiers pas dans les centres d'observation y côtoient, à dater de 1951, les éducateurs qui sont également à la recherche d'une référence identitaire et dont la mission est de "réformer" les méthodes éducatives. Ces éducateurs ont des statuts variés.

Les surveillants, qui formaient la plus grande partie des personnels des anciennes colonies et maisons de correction, ont eu le choix : être maintenus dans le cadre pénitentiaire ou opter pour une intégration dans le corps des éducateurs. Peu nombreux à le faire, ils y gagnent sur le plan de la rémunération, mais gardent longtemps une méfiance à l'égard des éducateurs formés par Vaucresson qui, " eux, ne savent pas ce qu'est le concret ». Ils furent quelquefois un frein au travail de ceuxci et à celui des psychologues.

D'autres éducateurs recrutés sous contrat, ayant une certaine expérience de vie, sollicitent le savoir "psy". Des instituteurs avaient été affectés, dès avant 1939, dans certains établissements. D’autres le furent durant la guerre, le plus souvent pour échapper au STO (service de travail obligatoire). Ils possèdent souvent des diplômes équivalents à ceux des psychologues et ont été mis à contribution pour la passation des tests. Parfois d'ailleurs, en attendant qu'un poste de psychologue soit vacant, des psychologues se font engager comme éducateurs. Parfois certains éducateurs, ayant acquis les diplômes nécessaires, demandent un tel poste, comme le statut le leur a permis de 1946 à 1981. De ce fait, l'élucidation des places propres des personnels éducatifs et des psychologues engendre parfois des rapports de force. 
Les contacts entre éducateurs et psychologues se font lors des réunions d'orientation technique ou lors des réunions de synthèse pendant lesquelles chacun apporte son point de vue. Peu de relations entre eux, sinon, au centre d'observation de Savigny, à l'occasion du repas, où d'ailleurs les psychologues restent groupés à la même table. Les représentations qu'ils ont les uns et des autres n'incitent sous doute pas à nouer des échanges.

Les psychologues sont parfois considérés comme originaux, au langage incompréhensible. Ils donnent l'impression d'appartenir à un monde clos dont l'entrée était interdite aux éducateurs. Ainsi un éducateur, qui a demandé à parler au docteur Mâle d'un adolescent qui avait de gros problèmes dans le groupe, s'est entendu dire par la psychologue qu'il n'avait rien à faire avec le psychiatre et qu'il aille s'occuper de son groupe.

Pour les éducateurs, de même que pour les professeurs techniques, les psychologues semblent "protégés" dans leur bureau. Ils ont des horaires confortables (bien qu'ils aient fait l'objet de notes de service pour leur retard ou leur absence) et travaillent en quelque sorte en libéral, alors que les éducateurs sont "au charbon", ont à supporter la violence d'un groupe et sont astreints à une présence du soir et du week-end. Ils estiment de plus qu'il y a un décalage entre les façons de percevoir les situations et que les psychologues méconnaissent la réalité.

L'ouverture des consultations, à la fin des années cinquante, va modifier les relations et permettre une forme, au moins institutionnelle, d'un travail assumé en commun, où la parole pourrait être partagée. En particulier, l'organisation de la prise en charge des jeunes donne une nouvelle place au psychologue. Il est présent lors de l'entretien d'accueil du sujet et de sa famille ; il prend connaissance de l'origine de ses difficultés dans ses différents lieux de vie; il entend son appréciation des faits ayant amené l'ordonnance d'une mesure ; il participe aux hypothèses relatives au cadre éducatif dont il a besoin.

La réflexion sur la présence des psychologues dans les institutions d'éducation sera indirectement favorisée par la création du service de la recherche à Vaucresson. En effet, l'une des premières enquêtes, menée à partir de 1958, sur 500 jeunes délinquants, réunira épisodiquement, puis 
(49) Voir les différents numéros des Annales de Vaucresson, qui publieront ces recherches et en particulier « L'internat de rééducation ", dirigé par J. Selosse en collaboration avec M. Jacquey, M.-Th. Mazerol, P. Segond, Vaucresson, éd. Cujas, 1972. de façon plus régulière, un certain nombre de psychologues de province qui y seront associés. Les psychologues affectés à cette recherche feront connaître aux personnels des institutions éloignées une pratique qui est aussi d'écoute, de compréhension. Enfin, il n'est sans doute pas sans signification que les premières institutions de l'Éducation surveillée, Saint-Maurice et Émancé, ayant souhaité la présence d'un psychologue, soient justement celles qui avaient été choisies comme lieu d'étude pour une recherche sur les internats ! ${ }^{(49)}$

Mais c'est seulement au début des années soixante-dix, avec l'élargissement des options éducatives, que se produit une innovation d'importance, lorsque les centres d'observation s'ouvrent à la " prise en charge globale » et que des postes de psychologues sont créés dans les institutions éducatives. Ce projet ancien n'avait pas recueilli une totale adhésion. Toutefois, l'ouverture de ces postes dans les institutions publiques d'éducation surveillée (IPES) amplifie la dispersion et pose des problèmes techniques, en particulier celui d'un exercice solitaire dans des établissements pas toujours très proches d'une ville universitaire. Il a été proposé que le temps soit partagé entre l'internat et une consultation ou un centre d'observation proche, et que ces postes soient occupés par des psychologues ayant deux ou trois ans d'exercice professionnel spécialisé au niveau de l'inadaptation juvénile (motion des psychologues au congrès syndical de 1968).

Cette forme inédite de travail oblige à penser différemment les modes d'exercice. Elle constitue une faille dans l'image d'un psychologue protégé par un rituel d'examen, même si ce rituel s'est assoupli. Il va s'agir, d'une part, à partir du décodage du vu/entendu/ressenti, d'évaluer les retombées éventuelles du projet éducatif et thérapeutique, de telle sorte que le passage dans l'institution éducative restitue aux jeunes une meilleure appréhension de l'acte, de la situation et, en cassant le répétitif, prenne place dans leur histoire ; d'autre part, de légitimer une "présence" rassurante auprès des personnels, ce qui soulève de tout autres interrogations.

Un certain flou s'installe. Les psychologues, majoritairement recrutés sous contrat, présentent des visées différentes selon leur formation universitaire et des expériences très variées. De surcroît, ils sont pris dans un discours institutionnel réducteur de leur spécificité et semblent par- 
fois, individuellement, ne plus savoir formuler ce qu'il en est de leur place et du cadre de leur travail, travail dont la définition, la délimitation sont toujours à recommencer en fonction de la rotation rapide des éducateurs et des responsables. Les psychologues, en raison du petit nombre de postes offerts, sont stables dans l'établissement et témoins de son histoire. Ils ne quittent l'institution que pour exercer dans le privé, enseigner à l'université ou s'installer derrière un divan.

Dans le même temps, les éducateurs et les juges des enfants ont acquis une culture en sciences humaines. Ils ont démythifié le rôle du psychologue (tout en méconnaissant parfois sa fonction réelle). L'évolution des sciences, la propagation des théories freudiennes dans la population ont eu une incidence sur une demande multiforme. Si les psychologues ne sont pas remis en cause, les exigences à leur égard en sont renforcées.

Il est à remarquer que là aussi une menace semble planer. Certains responsables vont opter pour l'engagement de psychologues vacataires, s'assurant ainsi que "leur" psychologue correspondra à leur idée. Ils marquent, de façon plus ou moins manifeste, que les psychologues sont dépendants de choix exprimés dans des lieux qui sont principalement ceux de la pratique d'autres. Mais ils sont aussi à l'origine de controverses qui affectent les relations professionnelles et rendront plus aléatoires un suivi individuel et un travail institutionnel dans ce contexte.

\section{En conclusion, le tournant des années soixante-dix}

Mai 1968 marque la fin d'une période et engendre une grande remise en question, un sursaut d'une exceptionnelle ampleur. Des assises nationales des personnels de l'Éducation surveillée, organisées dès juin 1968 à Vaucresson, rassemblent des éducateurs, des juges des enfants, des avocats, des professeurs d'université, des chercheurs du CNRS (avec une percée conséquente des sociologues et des psychosociologues), des éducateurs du secteur associatif, des psychiatres, des psychanalystes... Les réunions se multiplient. Des comités, des commissions se mettent en place, telle la commission tripartite (magistrats de l'administration centrale, représentants des personnels, personnalités de l'extérieur), qui échangent sur deux années. 
(50) Voir, en particulier, la loi 70-1320 du 31 décembre 1970 relative aux mesures sanitaires de lutte contre la toxicomanie.

(51) Ronald Laing a fondé, en 1965, Kingsley Hall, lieu de vie qui sera à la pointe du mouvement anti-psychiatrique. L'expérience de Neill à Summerhill, celle de Bettelheim à l'école orthogénique de Chicago, "milieu thérapeutique total", étaient connues. L'école expérimentale de Bonneuil, “institution éclatée”, sera fondée par Robert Lefort et Maud Mannoni en 1969. François Tosquelles a fait plusieurs interventions sur l'analyse institutionnelle à Vaucresson...
Durant les années soixante et soixante-dix, l'évolution de la manifestation des troubles, entre autres l'usage de toxiques, ${ }^{(50)}$ les nouvelles formes de violence, la déviance de plus en plus précoce de mineurs, ont nécessité une adéquation des pratiques, un partage d'outils conceptuels et l'invention d'autres espaces thérapeutiques.

Ainsi, entraînés par le tourbillon dénonciateur de la différence, soit hiérarchique, soit de savoir, les psychologues semblent avoir mis entre parenthèses le souhait d'unification de la profession. Portés par la montée des courants de l'antipsychiatrie, des thérapies institutionnelles, des mouvements alternatifs, ${ }^{(51)}$ certains semblent tentés par un univers du "même" et sont prêts à abandonner toute spécificité.

Il est vrai aussi que, au centre de Vaucresson, depuis 1966, une formation de longue durée (sur une année) animée à la fois par des sociologues, des psychosociologues, des psychologues et des psychanalystes - Jean Maisonneuve, Gilles Ferry, Françoise Martinier, Marie-Thérèse Mazerol, Joseph Villier... - avait été ouverte indifféremment aux psychologues et aux éducateurs exerçant en milieu ouvert ou appelés à prendre des responsabilités. Elle comprenait du " travail de groupe " et du " contrôle individuel ». Pour certains, cette expérience devait aboutir à une reprise d'études - de psychologie surtout - voire à une démarche analytique.

Deux pôles de discussion se dessinent :

- l'un commun aux deux professions, à savoir l'écrit, le secret professionnel. Le problème de l'écrit a été traité plus haut. Il est resté vivace, donnant lieu, en 1970, au Manifeste des cent qui était essentiellement le fait des personnels éducatifs. Des échanges, parfois acerbes, s'échangent sur l'opportunité de donner le rapport à lire au jeune et à sa famille.

- l'autre spécifique, la fonction de thérapeute, ce qui débouche sur une autre prise en considération du sujet confié à l'Éducation surveillée.

Concernant le problème, jugé essentiel de la psychothérapie, une sous-commission l'aborde sous deux aspects étroitement associés :

- les échanges sur la psychothérapie individuelle. Ils étaient assez avancés et avaient donné lieu à publication, d'une part par le docteur Mâle en 1966, dans les Annales de Vaucresson, d'autre part par le docteur Gonin sur son expérience au centre d'observation de Collonges. Une commission 
devait continuer à se réunir jusqu'en fin d'année 1971. ${ }^{(52)}$

- la considération d'une dimension thérapeutique de la "présence" dans ce "contexte" des institutions de l'Éducation surveillée, portée par la diffusion des avancées de la psychothérapie institutionnelle sous ses formes les plus variées (F. Tosquelles, J. Oury, E. Goffman, J. Pain), avec des références aux expériences de C. Freinet, F. Deligny, M. Mannoni...

Dans le même temps, des discussions s'engagent sur "l'analyse systémique » à l'Éducation surveillée, introduite en 1973 par Siegi Hirsch. Un intense travail de recherche et de traduction de textes américains et italiens est mené par Georges Durand, Pierre Segond et Christiane Chirol, qui participent à la création de la première consultation familiale de l'Éducation surveillée, rue Sedaine à Paris. Il donne lieu à de nombreux groupes de réflexion, de formation, d'inter-contrôle, relayés en province par des actions/recherches, en articulation avec des équipes de thérapie familiale tant en France qu'à l'étranger.

Malgré les résistances de certains responsables qui assimilent cette approche au comportementalisme, cette expérience, marquée non plus par la seule souffrance individuelle mais par les effets des interactions familiales, de la filiation, de la construction/destruction du lien, est accueillie avec intérêt par un certain nombre de cliniciens, d'éducateurs, de magistrats. Elle posera par ailleurs la question du paradoxe de " l'aide contrainte ".

Sur un autre plan encore, une expérience en consultation, en association avec le groupe Censier de sociopsychanalyse, fait l'objet d'une publication cette même année 1976. Il est à noter que c'est à partir de Censier (Paris VII), en 1968, que professeurs, étudiants et travailleurs sociaux (au sens large du terme) se rencontrent. De leurs discussions résultera l'aménagement d'un cursus de formation sous l'égide de professeurs, psychanalystes par ailleurs (parmi eux F. Gantheret, J. B. Pontalis, P. Fédida, O. Avron, Cl. Revault d'Allones, mais aussi G. Lapassade...). C'est dire la multiplicité des théories "visitées" ! Chacune enrichit les autres, coexiste avec les autres, ou est abandonnée.

Début 1976, ${ }^{(53)}$ un groupe de psychologues - Danièle Epstein, JeanMichel Labadie, Jean-Pierre Legeay, Annie Pignarre, Anne Raguin, Lau-

(52) Ainsi qu'en témoigne un compterendu dactylographié d'une réunion tenue le 2.10.1971 sur La psychothérapie dans le cadre de l'Éducation surveillée, durant laquelle se sont exprimés MM. de Thévenard, Selosse, Villier, Mmes Apellaniz, Duchier, Gaudin, les docteurs Bérouti, Martinier, Robin, Roumajon et Stork. À signaler également un rapport du docteur Martinier sur Pratique de la psychothérapie dans le cadre de l'Éducation surveillée, de 1972.

(53) Il est à remarquer, une fois encore, qu'un changement social donnant lieu à une loi nouvelle, ici la loi du 5 juillet 1974, fixant à 18 ans l'âge de la majorité, bientôt suivie par le décret du 18 février 1975 sur les modalités de mise en œuvre d'une action de protection judiciaire 
en faveur des jeunes majeurs, a des incidences immédiates sur l'action conduite à l'Éducation surveillée.

(54) Sans doute est-ce facilité par le rôle de passerelle joué par certains chercheurs de Vaucresson, Béatrice Koeppel, Marie-Thérèse Mazerol, Pierre Segond, Joseph Villier, qui ont une expérience première de la pratique dans les services de l'Éducation surveillée. Ceci est à noter, car l'une des qualités de l’Éducation surveillée, puis de la Protection judiciaire de la jeunesse, aura été sa curiosité et sa capacité de prendre en compte, souvent dans l'immédiat, les interrogations sur l'émergence de savoirs et d'organiser débats, commissions, stages, pour juger de leur pertinence dans ce cadre. rence Sanson.... - lancent une interrogation sur l'identité et les fondements de la pratique des psychologues. Il leur semble nécessaire de cesser de se questionner sur la profession en référence au judiciaire et à l'éducatif - donc de sortir du cadre institutionnel et du système d'échanges "vaucressoniste" habituel - et de créer des réseaux, des lieux, pour engager un autre cheminement. Des textes sont rassemblés, des groupes de travail constitués, afin de construire, lors d'une rencontre générale, un schéma de travail.

Une journée de synthèse regroupe, à Vaucresson, près de la moitié des psychologues (ils sont 150 à ce moment-là). Très rapidement des conflits d'école se font jour. Trois groupes se constituent afin de poursuivre la réflexion de façon indépendante. Même s'ils provoquent des remous, parfois des indignations, certains textes, en particulier ceux des lacaniens, contribuent à donner un nouveau souffle à la profession en questionnant le champ théorique et en posant un regard nouveau sur les méthodes de travail avec le sujet. Il peut paraître évident que cette considération du sujet soit prévalente dans ce métier, mais la question pouvait se poser à la lecture de quelques entretiens inquisiteurs et d'examens aux conclusions moralisantes, voire sentencieuses.

Avec des flux et des reflux, le désir de s'interroger à nouveau sur l'identité, sur les fondements de la profession dans le cadre judiciaire et institutionnel, sur le repérage du projet d'écoute et de compréhension de toutes les structures psychiques « en ce qu'elles fondent l'intra et l'intersubjectif ", est certes enrichi par les courants du moment. Mais il se maintient depuis lors et incite les psychologues à effectuer des travaux sur la pratique, les méthodes, les théories. Il s'appuie en cela parfois sur une organisation régionale, parfois sur le Service des études de Vaucresson, parfois sur le Centre de recherche interdisciplinaire de Vaucresson, ${ }^{(54)}$ qui conservent le souci de renforcer les interrelations nécessaires entre pratique professionnelle et recherche et de faire bénéficier les praticiens des apports les plus récents des sciences humaines.

Cette effervescence a finalement atteint un but, celui de donner conscience aux psychologues de l'Éducation surveillée qu'ils exercent une profession originale, bien que toujours à définir par rapport à la loi, à l'administratif, à l'éducatif, mais aussi au modèle théorique, à l'inscription 
dans un groupe, voire une école. Mais ils se savent appartenir aussi, de ce fait, à une profession plus vaste, grâce à la reconnaissance qui leur vient de l'extérieur sous forme de demandes d'intervention variées, soit pour la formation des personnels, soit dans le cadre de réunions portant sur des études de situation ou sur l'élucidation de difficultés rencontrées par d'autres professionnels, soit lors de supervisions ou de séminaires, soit parce qu'ils enseignent à l'Université.

Toutefois, pour les psychologues, le primordial du travail clinique reste la rencontre de l'enfant et de l'adolescent. Et c'est particulièrement là que la perplexité reste grande. En effet, tout au long de leur pratique, les psychologues se sont heurtés à une butée, qui est celle justement d'une spécificité de la clinique dans le champ judiciaire/éducatif. Butée non pas quant à la constitution de savoirs cohérents - le psychologue a montré qu'il pouvait contribuer à fournir l'information "scientifique" et clinique nécessaire - mais butée quant à une application de ces savoirs auprès de ces jeunes sans repères, ces "cas-limites", à " une espèce de carrefour de l'indétermination ", ${ }^{(55)}$ confrontés à une loi qu'ils défient quand ils ne l'ignorent pas. Le sens de l'ordonnance prise par le juge leur échappe : ils ne savent pas pourquoi ils sont là. Ils ne sont pas fous. Il a été dit souvent qu'ils transgressaient la loi. Or l'acte qui les conduit devant le juge pourrait représenter un appel inconscient à cette "loi", celle portée par le "père" et qui institue une limite structurante.

Mais surtout, pour beaucoup d'entre eux, les mots manquent qui leur permettraient éventuellement d'approcher la signification de leur acte. Or le sujet n'existe que dans et par le langage. Il naît soumis à un ordre symbolique, il « est parlé » avant qu'il ne parle et les manifestations de son inconscient débordent ses énoncés.

Que peut faire le psychologue face à ces jeunes qui présentent un certain désarroi dans les divers mouvements d'identification, pour lesquels les "facteurs verbaux et spéculatifs sont davantage atteints que les aptitudes pratiques, concrètes et techniques ", et qui ont des difficultés d'abstraction, de symbolisation et d'écriture, accompagnées d'une incapacité à se concentrer ? Bloqués pour la plupart dans leur parole, ils s'expriment plus volontiers par l'acte, l'acte sans mot. Ces jeunes, vivant dans la misè-

(55) André Green, Cent ans après, op. cit., p. 145. 
re existentielle, au moi insuffisant, dont l'intelligence est souvent blessée, troublée, se sont vus rejetés des processus d'éducation "normaux" et ont été "pris" très tôt dans une représentation imaginaire. Si certains rentrent dans les catégories bien explicitées de conduites relevant de la psychiatrie ou d'une action éducative, voire thérapeutique, d'autres nécessitent une toute autre voie de compréhension et d'accompagnement. Les psychologues se sont centrés sur l'opportunité d'un espace clinique, voire thérapeutique pour eux. Mais, depuis les recherches concernant le fruste, en passant par le borderline, l'incasable, le désaffilié, le "vrai" délinquant, l'immigré interdit de mémoire et d'histoire, le jeune marginal, paumé, morose, difficile, très difficile..., il n'a guère été "pensable" d'atteindre une voie spécifique de compréhension et de traitement. Ils "résistent". Et les psychologues continuent à interroger les lieux épistémologiques et à rêver d'une théorie qui ouvre sur d'autres possibles de la clinique. 